\title{
Conditioning Gaze-Contingent Systems for the Real World: Insights from a Field Study in the Fast Food Industry
}

\author{
Melanie Heck \\ University of Mannheim \\ Mannheim, Germany \\ melanie.heck@uni-mannheim.de
}

\author{
Janick Edinger \\ University of Hamburg \\ Hamburg, Germany \\ edinger@informatik.uni-hamburg.de
}

\author{
Christian Becker \\ University of Mannheim \\ Mannheim, Germany \\ christian.becker@uni-mannheim.de
}

\begin{abstract}
Eye tracking can be used to infer what is relevant to a user, and adapt the content and appearance of an application to support the user in their current task. A prerequisite for integrating such adaptive user interfaces into public terminals is robust gaze estimation. Commercial eye trackers are highly accurate, but require prior person-specific calibration and a relatively stable head position. In this paper, we collect data from 26 authentic customers of a fast food restaurant while interacting with a total of 120 products on a self-order terminal. From our observations during the experiment and a qualitative analysis of the collected gaze data, we derive best practice approaches regarding the integration of eye tracking software into self-service systems. We evaluate several implicit calibration strategies that derive the user's true focus of attention either from the context of the user interface, or from their interaction with the system. Our results show that the original gaze estimates can be visibly improved by taking into account both contextual and interaction-based information.
\end{abstract}

\section{CCS CONCEPTS}

- Human-centered computing $\rightarrow$ Field studies; Gestural input.

\section{KEYWORDS}

eye tracker calibration, gaze interfaces, eye tracking

\section{ACM Reference Format:}

Melanie Heck, Janick Edinger, and Christian Becker. 2021. Conditioning Gaze-Contingent Systems for the Real World: Insights from a Field Study in the Fast Food Industry. In CHI Conference on Human Factors in Computing Systems Extended Abstracts (CHI '21 Extended Abstracts), May 8-13, 2021, Yokohama, Japan. ACM, New York, NY, USA, 7 pages. https://doi.org/10. $1145 / 3411763.3451658$

\section{INTRODUCTION}

The amount of information created worldwide has been growing exponentially in the past ten years [31], leading Amazon to develop sophisticated algorithms that analyze customers' transactions and recommend products that are relevant to them [20].

Permission to make digital or hard copies of all or part of this work for personal or classroom use is granted without fee provided that copies are not made or distributed for profit or commercial advantage and that copies bear this notice and the full citation on the first page. Copyrights for components of this work owned by others than the author(s) must be honored. Abstracting with credit is permitted. To copy otherwise, or republish, to post on servers or to redistribute to lists, requires prior specific permission and/or a fee. Request permissions from permissions@acm.org.

CHI '21 Extended Abstracts, May 8-13, 2021, Yokohama, Japan

(c) 2021 Copyright held by the owner/author(s). Publication rights licensed to ACM ACM ISBN 978-1-4503-8095-9/21/05 . .\$15.00

https://doi.org/10.1145/3411763.3451658
Facebook issues targeted advertisements based on the users' browsing behavior [5]. These approaches channel the users' attention and make the experience more engaging. The more information about a user is available, the better the content can be tailored towards their needs and preferences. On public terminals where user interactions are generally short, information about individual users is scarce. Eye tracking has thus be explored as a technique to quickly identify user preferences based on a minimal amount of user interaction $[1,6,8,14,18,19,22,27,29,30,34,35,38]$. This, however, requires robust gaze estimation that works without prior person-specific calibration and is able to cope with changing lighting conditions, head movement, users diverting their gaze, and other contextual influences.

State-of-the-art research has mostly focused on the advancement of robust gaze estimation methods [2, 7, 15, 32, 33, 36]. Yet, these approaches are not readily accessible to industry participants who might not have the technical knowledge to implement their own eye tracking system. Commercial screen-based eye trackers produce readily usable gaze data and can be integrated into existing applications with minimal technical expertise. However, they require prior calibration and a relatively stable setup. We thus propose recording users' gaze data with ready-to-use eye tracking systems, and then a posteriori re-calibrate the data. In an on-site experiment in a fast food restaurant, we collect the gaze data of 26 authentic customers while operating a self-order terminal. We then evaluate four implicit interaction- and context-informed re-calibration strategies and test whether they can improve the quality of the collected gaze data. The approach can be applied to any commercial or camera-based gaze tracker output and is robust to distractions.

\section{RELATED WORK}

Commercial eye trackers are highly accurate, but require personspecific calibration. Since this makes them impractical for spontaneous interactions, they are mainly used in lab studies.

Evidence for the use of eye tracking in a real-world scenario can be found in psychological studies, where mobile eye trackers are an established technique to gather insights into the determinants of people's attention [21, 23]. Market research has started using eye glasses in in-store experiments to analyze customers' attention to products [9, 11, 16, 24, 37] and advertisements [25] while shopping. While these studies use eye tracking in a real-world scenario, participants are aware of the experimental setup and are subjected to person-specific eye tracker calibration. Since head-mounted devices are employed, environmental conditions such as lighting or eye occlusion do not have to be accounted for.

Classical point-to-point calibration requires the user to fixate a succession of usually five or nine points on the screen [26]. In a 
real-world application, such an approach would be too intrusive. Guestrin and Eisenmann [12] thus propose using multiple cameras and light sources to reduce the number of calibration points. Yet, the single-point calibration model is significantly less accurate than the traditional five-point calibration. Using a gamification approach, Renner et al. [28] visually and acoustically augment a dragonfly in a virtual reality classroom. Assuming that the object draws the user's attention, its position is aligned with the recorded gaze data. Pursuit calibration [26] exploits the large number of reference points of a moving target. It calculates the correlation coefficient of the recorded gaze data and the coordinates of a moving object. If it exceeds a predefined threshold, the user can be assumed to have followed the moving object and the data is used for calibration. The method has been shown to be robust to the user being unaware of the procedure, as well as to interruptions during the process. In a recent regression-based variation of the pursuit calibration, Drewes et al. [10] achieve an accurate calibration within 2 seconds using an area of calibration of a mere $4^{\circ}$ visual angle. However, the accuracy is still lower than for the traditional nine-point calibration.

Saliency correlation makes use of the fact that certain areas in an image are more salient than others due to low-level image features such as color and contrast, and thus attract human attention. By assuming that a strong correlation between these areas and a user's gaze exists, eye trackers can be calibrated. Chen and Ji [7] use a Bayesian Network for probabilistic model-based gaze estimation. They then update the calculated probability by combining it with the visual saliency map of the displayed image. Sugano et al. [32] present a similar approach for appearance-based gaze estimation. Saliency maps are treated as probability distributions of gaze points. Using a Gaussian process regression, the eye images are mapped to the estimated gaze points. Yet, low-level features do not account for people allocating more attention to certain features such as faces or specific shapes. Alnajar et al. [2] thus calibrate the gaze data of a new user by mapping it to the gaze data recorded from other humans using K-closest points fitting. The approach presupposes that users who look at the same scene have similar gaze patterns. Wang et al. [36] train deep learning models to learn salient features implicitly. Model- or feature-based eye trackers can then be calibrated by minimizing the difference between the saliency-based gaze prediction and the recorded gaze distribution.

Assuming that users look at the cursor when performing a mouse click, interaction-informed calibration aligns the gaze with the screen position of interaction elements. Sugano et al. [33] create a new training sample for appearance-based gaze estimation whenever the user performs a mouse click. The input vector consisting of head pose and eye image is then labeled with the cursor coordinates. In order to avoid the creation of false labels, Huang et al. [15] feed new training sample to the model only if the estimated gaze coordinates match the coordinates of the interaction element.

Existing approaches are tightly coupled with gaze estimation and thus require complete control over the estimation method. In contrast, we need a technique that can be used to a posteriori calibrate gaze data after its collection with an arbitrary eye tracker.

\section{IN-STORE EXPERIMENT: EXPLORATORY STUDY}

As a testbed for exploring the issues associated with eye tracking in a real-world setting, we integrated a screen-based eye tracker into a self-order terminal placed at a fast food restaurant situated in an industrial area (see [13] for a detailed description of the system). Our objective was to find out whether there are additional factors that negatively impact the conclusiveness of gaze data when recording them in the field. In order to test the setup with a realistic application, we replicated the brand's self-order system, while logging all customer interactions and recording their gaze data.

\subsection{Participants}

The study was conducted with 26 participants (17 male, mean age $=40.04, \sigma=20.00$ ) who were randomly recruited upon entering the restaurant. Most subjects were industrial workers who visited the restaurant during their lunch break or after work. The restaurant had not yet installed any self-order terminals. Thus, none of the participants had previously ordered on a terminal of the brand. Subjects were informed that they were participating in a scientific study, but were unaware that their gaze was being recorded. They were reimbursed with a dessert coupon valid for that day.

\subsection{Apparatus}

The self-order terminal was realized with a 23.6 inch iiyama touch monitor (1920 x 1080 pixels) mounted to a counter (height: 43 inches) at a distance of 18 inches off the front edge, and at an angle of $60^{\circ}$ to the tabletop. This setup was found to deliver reliable gaze data for users of different physique and posture. Figure 1 depicts the experimental setup and used material. The software ran on a $2.5 \mathrm{GHz}$ Intel Core i7-6500 HP PC with 8GB RAM. For gaze data collection, a screen-based Tobii Pro X3-120 eye tracker with a sampling frequency of $120 \mathrm{~Hz}$ was mounted to the display.

\subsection{Procedure}

Participants were asked to place their order at the terminal. A research assistant stood by to provide help if a customers had difficulties operating the system, but did not interfere otherwise.

An initial calibration was performed for each customer to align their gaze with the display coordinates. In order to provide a realistic experience, we applied an unobtrusive point-to-point calibration. The central calibration point consisted of an interactive button with the brand's logo. Customers had to press the button to initiate the system. This secured their attention to the screen. We then let the image of a burger move along the top of the screen from right to left, and then again along the bottom from left to right. Calibration data was collected in each of the four corners of the display. While the unobtrusive approach does not guarantee that the customer pays attention to the calibration stimulus, we deemed the increased usability compared to a 9-point calibration more important.

Upon the customer's selection of an item, the eye tracker started recording their gaze. It stopped when a navigation button was clicked. If the customer subsequently selected another product, the eye tracker resumed recording their gaze. The new gaze data was 


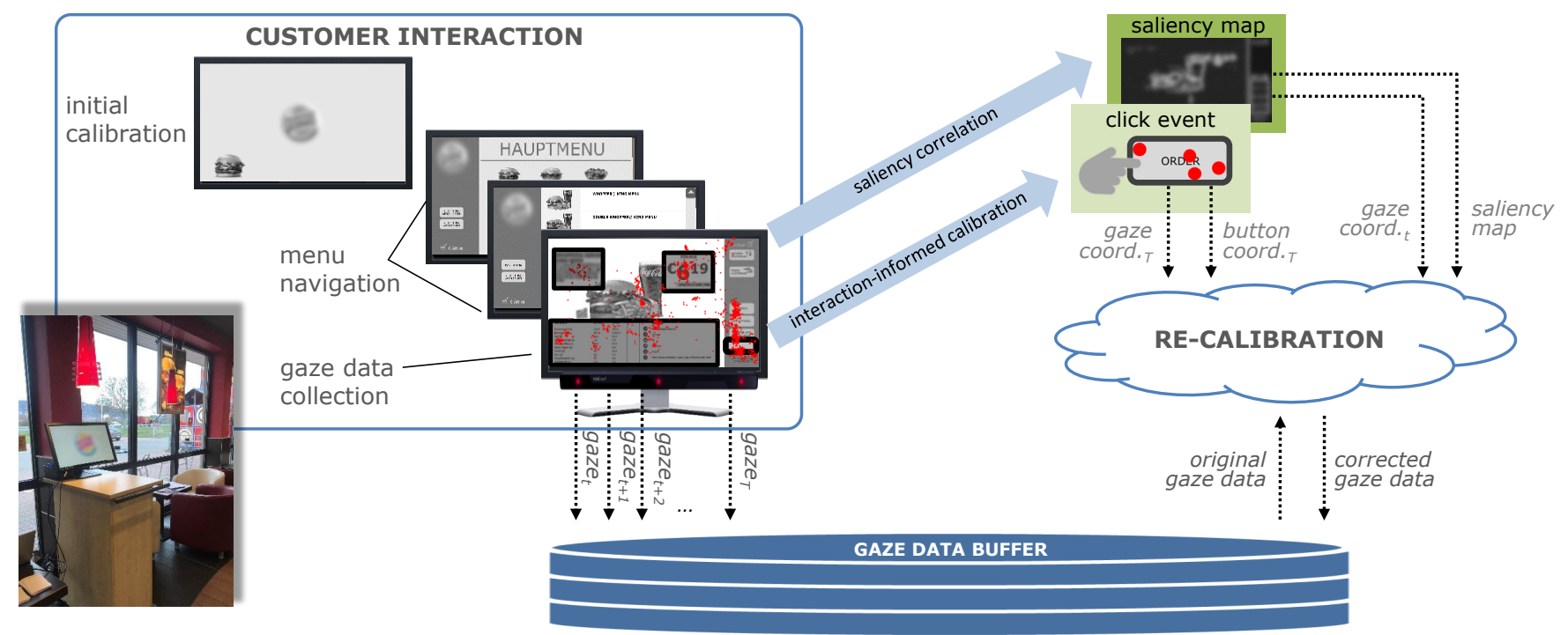

Figure 1: Application of interaction and context information to re-calibrate the recorded gaze data.

stored in a separate file. This allows to individually re-calibrate the gaze data recorded between any two user interactions.

\subsection{Data Analysis}

The collected data and observations from the interaction with the customers delivered valuable insights which may be relevant for the design of real-world eye tracking systems:

- Perceived intrusiveness of the eye tracker. Despite the clear visibility of the eye tracker's infrared light source, none of the participants consciously registered its presence.

Conclusion: The presence of an eye tracking device does not bias the user's interaction with the system.

- Gaze data quality. On average, 4281 gaze points were recorded per customer, but only $36 \%$ of the recordings delivered valid gaze data. Assuming a blink frequency of $4.5 \mathrm{blinks} / \mathrm{min}$ [4] and a blink duration of $150 \mathrm{~ms}$ [3], a mere $1.13 \%$ of invalid gaze data can be attributed to natural blink behavior. The remaining misses were caused by customers either being positioned outside of the detectable range of the eye tracker, or diverting their gaze in conversation and other distractions. Conclusion: The interaction with the system should be intuitive to avoid that users avert their gaze in search of assistance. The amount of displayed information should be sufficiently high to demand the user's undivided attention.

- Effectiveness of initial calibration. Gaze data validity was analyzed based on visual examination of the recorded gaze points. Accurate recordings evince an accumulation of gaze points around the selection buttons. Yet, most samples exhibit a clear horizontal and/or vertical shift of the gaze data. Our observations revealed that a major determinant of the low quality of the gaze estimates was the inability of the calibration to attract customers' attention. Customers often engaged in conversation with the instructor or other customers during calibration, or touched the screen in an attempt to skip the procedure.

Conclusion: Using the trajectory of moving objects that are not an integral part of the task does not attract the users' attention, and does not result in satisfactory calibration. The evaluations by Pfeuffer et al. [26] suggest that an initial calibration based on the pursuit approach is capable of coping with these issues: Data validity is ensured by pausing the calibration whenever the user is inattentive. A seamless integration of the calibration stimulus has been successfully demonstrated within multiple application contexts.

We analyzed the effect of four re-calibration strategies:

(1) Size-dominant saliency correlation (S1). This strategy compares the initial focus of attention to the largest salient element on the screen resulting from a snapshot of the user interface. We used the openCV implementation for static saliency detection to determine salient elements. The center point of the element with the largest radius is considered the true gaze coordinate. The offset is calculated as the difference between the true and the recorded gaze coordinate. It is applied to all gaze points of the current file.

(2) Interaction-informed calibration (S2). The coordinates of a clicked button are used to correct the initial calibration. The Re-calibration module calculates the offset between the screen coordinates of the button center and the recorded gaze data. The offset is then added to all gaze data that was collected for the current item.

(3) Focused saliency correlation (S3). If customers face the terminal from a moderately frontal position, large deviations are uncommon. We therefore test whether saliency correlation delivers more accurate calibration if the initial focus is determined as the center point of the salient element closest to the recorded gaze coordinate. We first applied the calibration in isolation (S3-1). Then, we tested whether the strategy 


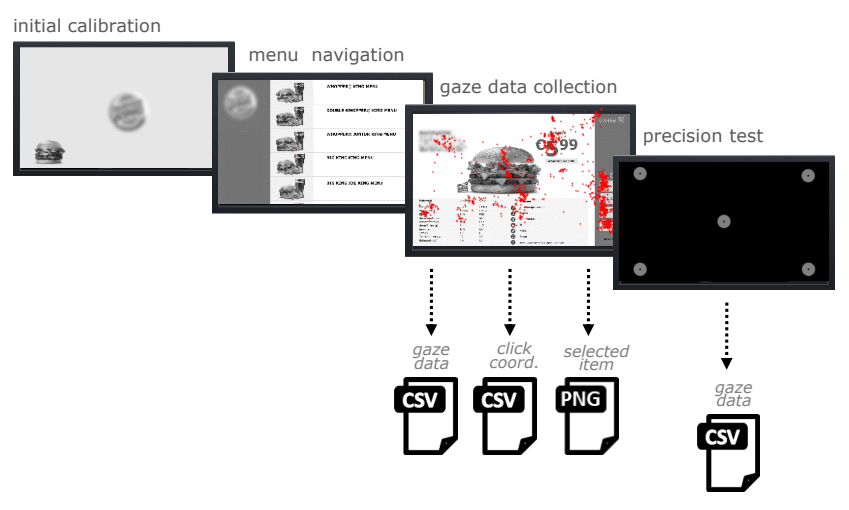

(a) Program sequence of the lab study order application.

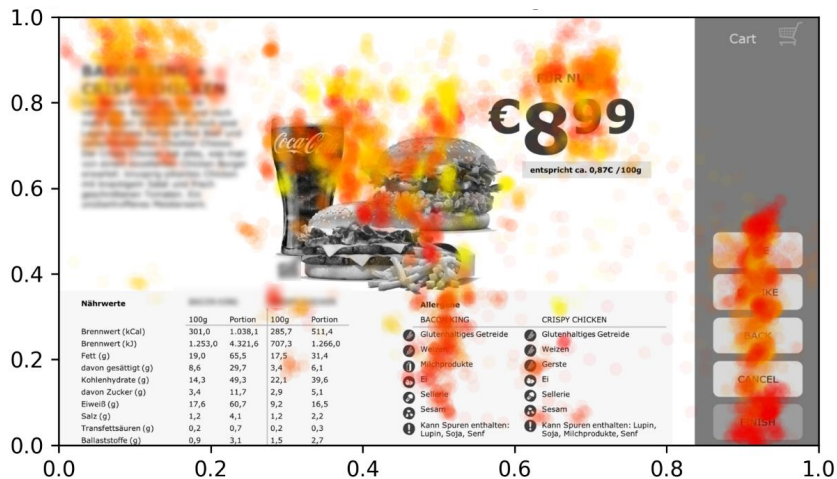

(b) Aggregated gaze allocation from all lab study participants.

Figure 2: Program sequence and gaze data output of the lab study.

delivers better results if applied to gaze data that has already been subjected to interaction-informed calibration (S3-2).

(4) Pixel-wise saliency correlation (S4). The user's initial focus is calculated as the mean coordinates of the first 20 valid gaze points that the eye tracker records. (Gaze points collected during the first second are usually influenced by the customer's attention to objects on the previous page, and are thus discarded.) The initial focus of attention is compared to the saliency map [17] of a snapshot of the user interface. We calculate the distance of the averaged gaze coordinates to the $10 \%$ most salient pixels on the interface (this parameter has to be adjusted according to the amount of background clutter). The pixel with minimum distance to the initial focus is considered the true gaze coordinate. The offset is calculated as the difference between the true and the recorded gaze coordinate. It is applied to all gaze points of the current file. We tested the calibration both in isolation (S4-1) and after a prior interaction-informed calibration (S4-2).

Figure 1 illustrates how the interaction and context data gathered during a user's interaction with the system is applied to re-calibrate the collected gaze data. S3 and S4 were tested both individually and in conjunction with interaction-informed calibration. Since S1 and $\mathbf{S} \mathbf{2}$ are based on absolute reference points, the prior application of another calibration would have no effect on the output.

Integrating a gaze data validation procedure into the order terminal would compromise the authenticity of the situation. Since a quantitative evaluation of the gaze data quality is therefore not possible, the data from the in-store experiment was analyzed based on visual examinations. The gaze patterns were compared to the assuredly accurate gaze distributions of users operating the system. These comparison data were collected in a controlled lab study.

\section{LAB STUDY: DETERMINATION OF TRUE GAZE DISTRIBUTION}

The benchmark for high-quality gaze data was established in a lab study. We applied a five-point calibration and calculated the precision of the data after a participant's interaction with the system.

\subsection{Method}

We collected gaze data of 8 members of our research group (6 male, mean age $=33.88, \sigma=11.28$ ). All were fully aware of the purpose of the study, but had not previously worked with the order software.

The study was performed in our research lab. The software ran on a $1.6 \mathrm{GHz}$ Intel Core i5-8250U Lenovo ThinkPad T480 with 8GB RAM. The graphical user interface was projected onto a 21.5 inch Samsung SyncMaster BX2240 monitor (1920 x 1080 pixels) which was positioned on a tabletop to mirror the setup of the in-store experiment. The same screen-based Tobii Pro X3-120 eye tracker that we used for the in-store experiment was mounted to the display.

Participants were asked to position themselves in front of the self-order terminal and navigate through the menu to place an order of their choice. A simplified version of the self-order application was used (see Figure 2a). After the initial calibration, participants could directly choose one out of five products which were chosen randomly from a pool of 20 items. As soon as the selected item was displayed, gaze data collection started. Similar to the full-fledged system, participants could press one out of five navigation buttons. The recorded gaze data, click coordinates, and selected item were each exported to a separate file. After participants pressed any selection button, the instructions for the precision test were displayed on the screen. They were asked to look at the center of five red circles that consecutively appeared in each of the five corners and in the center of the screen, while their gaze data was being recorded.

\subsection{Data Analysis}

After the experiment, we evaluated the precision of the recorded gaze samples to verify that they correspond to the true gaze distribution of a user while interacting with the self-order system. All data had a mean error below .5 degrees of visual angle and can thus be considered to accurately represent the user's gaze allocation. We then visualized the gaze data. Figure $2 b$ shows the aggregated gaze data of all participants. A clear pattern is visible, showing an increased accumulation of gaze points on the interaction buttons and product-specific information detailing the textual item description, price and allergens. 


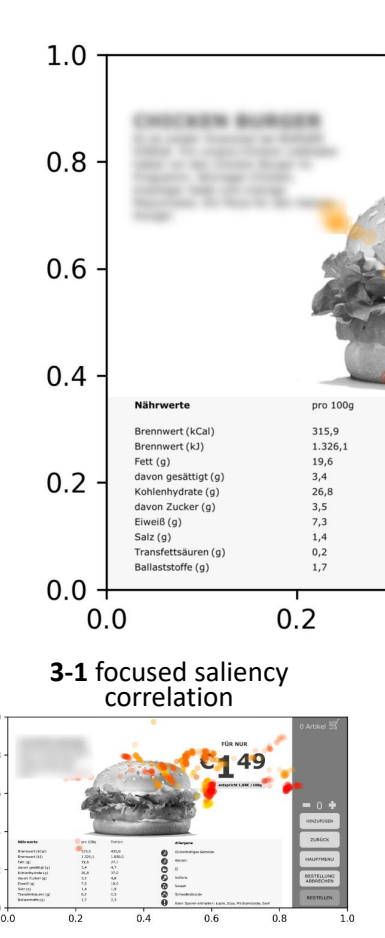

Initial calibration only

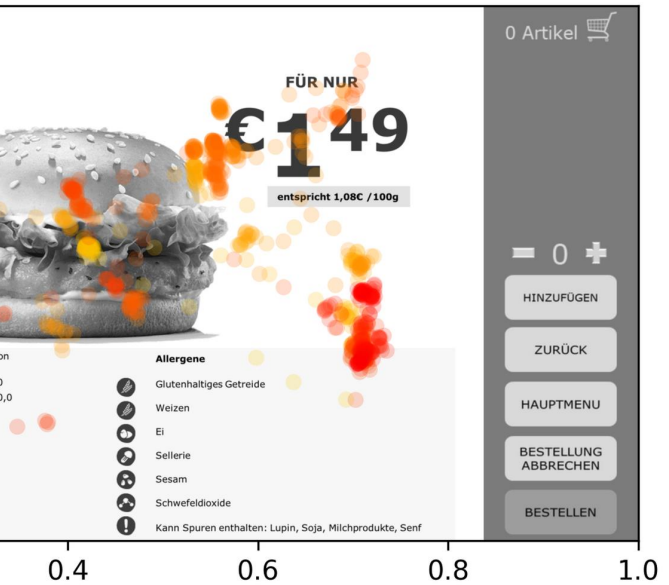

3-2 focused saliency + interaction-informed calibration

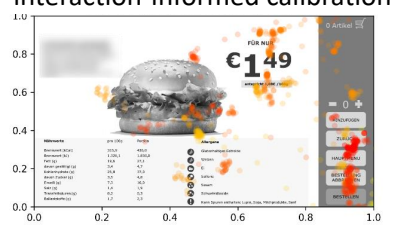

4-1 pixel-wise saliency correlation

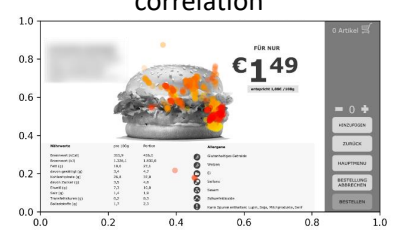

1 size-dominant saliency correlation

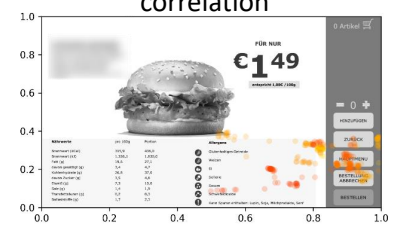

2 interaction-informed calibration

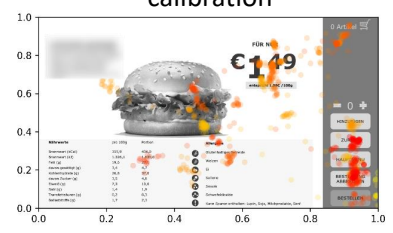

4-2 pixel-wise saliency + interaction-informed calibration

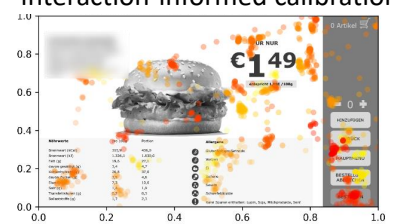

Figure 3: Exemplary gaze distribution of a customer from the in-store experiment after applying each implicit calibration.

\section{RESULTS}

We compared the gaze data from the in-store experiment to the typical gaze pattern that we identified in the controlled setup. A visual inspection was used to assess the validity of each calibration approach. Eight samples were excluded because the collected gaze data was too scarce to unambiguously determine whether calibration lead to a more reasonable gaze distribution. The remaining 18 samples were subjected to our visual analysis.

A representative gaze output is shown in Figure 3. Interactioninformed calibration delivers good results. Saliency correlations, in contrast, only lead to a visible improvement in a small amount of samples when applied in isolation, and the change was usually small. The size-dominant saliency correlation (S1) even shifted the initial calibration for the worse. We conclude that the mere size of salient objects does not necessarily determine the user's initial focus of attention. When applied after a prior interactioninformed calibration, the saliency-based calibrations performed visibly better $(\mathbf{S 3}, \mathbf{S 4})$.

Overall, the gaze distributions showed the most consistent patterns after applying focused saliency correlation in conjunction with interaction-informed calibration (S3-2). We conclude that users generally look at interaction buttons when clicking on them. Since, however, touch screen terminals have no visible pointer, the click coordinates are only an approximation of the user's true gaze. Our findings suggest that focused saliency correlation might be beneficial to further refine interaction-informed calibration. Yet, quantitative evaluations of a large number of data are needed to assess whether the improvement is significant. Figure 4 shows the number of samples in which each strategy resulted in an improvement or deterioration of the initial calibration. The results are mostly independent of the quality of the initial calibration (i.e., whether the input data is already close to the true gaze distribution).

\section{DISCUSSION}

We applied four re-calibration strategies to the gaze data from 26 authentic customers of a fast food restaurant, and compared their effectiveness. The calibration strategies derive a user's true focus of attention by correlating the gaze either with salient elements of the user interface, or with the coordinates of interaction events. Our results show that taking into account interaction information visually improves the quality of the original gaze data. While saliency information does not result in a better output when applied in isolation, it may be beneficial for more fine-grained calibration when applied after interaction calibration. Interaction and context information has been shown to be beneficial when directly integrated into model-based [7] or appearance-based gaze estimation [15, 32]. Our results allow us to be optimistic that calibration based on such information can also be successfully applied to already recorded gaze data. This will be useful to re-calibrate gaze data from commercial or third-party eye trackers where control over the gaze estimation is limited.

Since adding objective validity checks for the recorded gaze data would have compromised the authenticity of the experiment, we assessed the data quality based on visual examination. While 


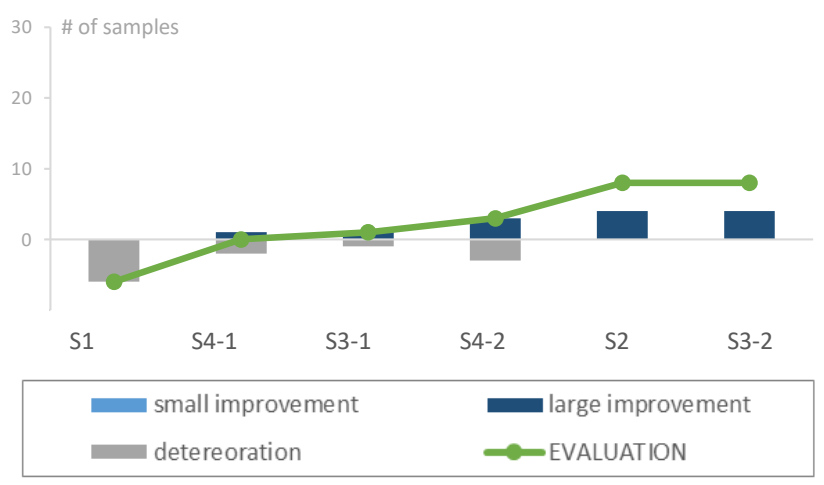

(a) good initial calibration

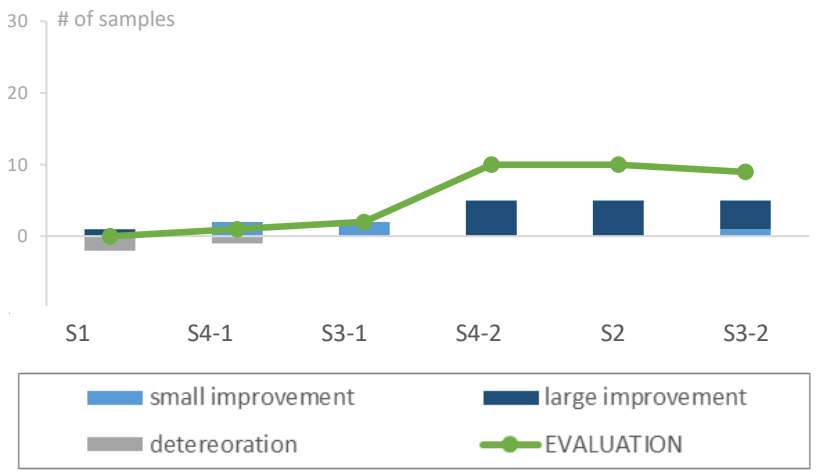

(c) poor initial calibration

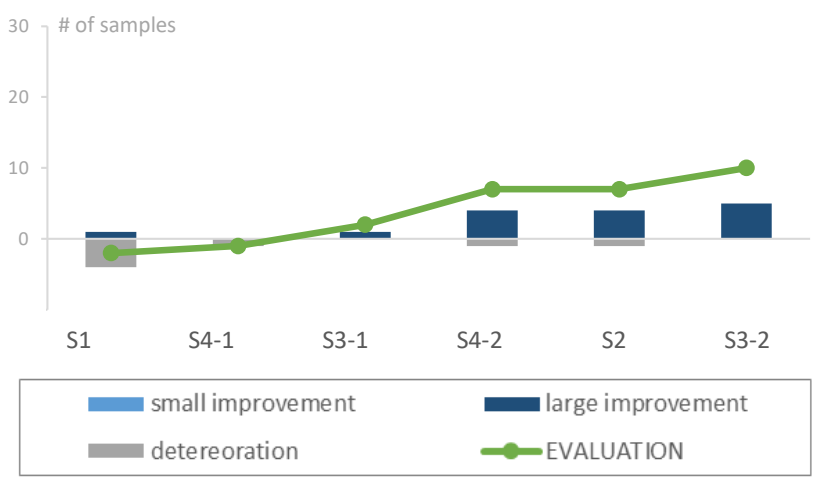

(b) acceptable initial calibration

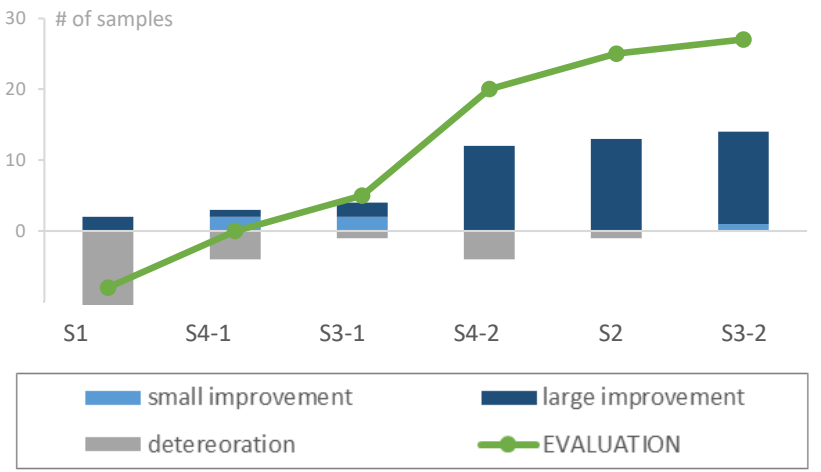

(d) overall evaluation

Figure 4: Effectiveness of the tested calibration strategies according to visual inspection. The plots show the number of samples in which each calibration strategy resulted in a small or large improvement, or in a deterioration of the original data. Large improvements were assigned higher weights in the overall evaluation by multiplying them with factor 2 .

our qualitative evaluation already suggests that the proposed recalibration strategies can effectively improve the quality of the gaze data, more work has to be put into their quantitative analysis. Our future work will thus focus on collecting gaze data in a controlled setting while users interact with the self-order terminal. We will use the collected gaze data to quantify the accuracy of gaze estimates under each calibration strategy. By testing the validity of each strategy under diverse conditions, we postulate that the results can be extrapolated to the in-store setting. Based on the quantitative results, we will thus propose a generalized procedure for unobtrusive eye tracker calibration in a real-world setting.

\section{REFERENCES}

[1] 2019. Combining Virtual Reality and Mobile Eye Tracking to Provide a Naturalistic Experimental Environment for Shopper Research. Journal of Business Research 100 (2019), 445-458. https://doi.org/10.1016/j.jbusres.2017.09.028

[2] Fares Alnajar, Theo Gevers, Roberto Valenti, and Sennay Ghebreab. 2013. Calibration-free gaze estimation using human gaze patterns. In Proceedings of the IEEE International Conference on Computer Vision (ICCV '13). IEEE, 137-144. https://doi.org/10.1109/ICCV.2013.24

[3] Simone Benedetto, Marco Pedrotti, Luca Minin, Thierry Baccino, Alessandra Re, and Roberto Montanari. 2011. Driver workload and eye blink duration. Transportation Research Part F Traffic Psychology and Behavior (2011), 199-208. https://doi.org/10.1016/j.trf.2010.12.001

[4] Anna Rita Bentivoglio, Susan B Bressman, Emanuele Cassetta, Donatella Carretta, Pietro Tonali, and Albert Albanese. 1997. Analysis of blink rate patterns in normal subjects. Movement Disorders 12, 6 (1997), 1028-1034.

[5] Anthony Bigornia. 2015. IBM/Facebook Partnership - Making Irrelevant Ads a Thing of the Past. https://www.ibm.com/blogs/insights- on-business/consumerproducts/ibmfacebook-partnership-making-irrelevant-ads-a-thing-of-thepast/

[6] Richard A. Bolt. 1981. Computer Graphics Volume 15, Number 3 August 1981. Computer Graphics 15, 3 (1981), 263-268. https://doi.org/10.1145/965161.806819

[7] Jixu Chen and Qiang Ji. 2011. Probabilistic gaze estimation without active personal calibration. In IEEE Computer Society Conference on Computer Vision and Pattern Recognition (CVPR'11). IEEE, Providence, RI, USA, 609-616. https://doi.org/10.1109/CVPR.2011.5995675

[8] Shiwei Cheng and Ying Liu. 2012. Eye-Tracking based adaptive user interface: Implicit human-computer interaction for preference indication. Fournal on $\mathrm{Mul}$ timodal User Interfaces 5, 1-2 (2012), 77-84. https://doi.org/10.1007/s12193-0110064-6

[9] Jesper Clement, Tore Kristensen, and Kjell Grønhaug. 2013. Understanding consumers' in-store visual perception: The influence of package design features on visual attention. Fournal of Retailing and Consumer Services 20, 2 (2013), 234-239. https://doi.org/10.1016/j.jretconser.2013.01.003

[10] Heiko Drewes, Ken Pfeuffer, and Florian Alt. 2019. Time- and space-efficient eye tracker calibration. In Eye Tracking Research and Applications Symposium (ETRA '19). ACM, Denver, CO, USA. https://doi.org/10.1145/3314111.3319818

[11] Kerstin Gidlöf, Richard Dewhurst, Kenneth Holmqvist, and Annika Wallin. 2013. Using eye tracking to trace a cognitive process: Gaze behaviour during decision making in a natural environment. Fournal of Eye Movement Research 6, 1 (2013), 1-14. https://doi.org/10.16910/jemr.6.1.3

[12] Elias Daniel Guestrin and Moshe Eizenman. 2006. General theory of remote gaze estimation using the pupil center and corneal reflections. IEEE Transactions on Biomedical Engineering 53, 6 (2006), 1124-1133. https://doi.org/10.1109/TBME. 2005.863952 
[13] Melanie Heck, Janick Edinger, and Christian Becker. 2019. Gaze-based product filtering: A system for creating adaptive user interfaces to personalize stateless point-of-sale machines. In 32nd Annual ACM Symposium on User Interface Software and Technology (UIST '19). ACM, New Orleans, LA, USA, 75-77. https://doi.org/10.1145/3332167.3357120

[14] Melanie Heck, Janick Edinger, Jonathan Bünemann, and Christian Beck. 2021. The subconscious director: Dynamically personalizing videos using gaze data. In 26th International Conference on Intelligent User Interfaces (IUI '21). ACM, College Station, TX, USA, 1-18. https://doi.org/10.1145/3397481.34506791

[15] Michael Xuelin Huang, Tiffany C.K. Kwok, Grace Ngai, Hong Va Leong, and Stephen C.F. Chan. 2014. Building a self-learning eye gaze model from user interaction data. In Proceedings of the 2014 ACM Conference on Multimedia (MM '14). ACM, Orlando, FL, USA, 1017-1020. https://doi.org/10.1145/2647868.2655031

[16] Rupert A. Hurley, Julie Christine Rice, Jerry Koefelda, Robert Congdon, and Andrew Ouzts. 2017. The role of secondary packaging on brand awareness: Analysis of 21 carbonated soft drinks in resusable shells using eye tracking technology. Packaging Technology and Science 30 (2017), 711-722. https://doi org $/ 10.1002 /$ pts. 2316

[17] Laurent Itti and Christof Koch. 2001. Computational modelling of visual attention. Nature Reviews Neuroscience 2, 3 (2001), 194-203. https://doi.org/10.1038/ 35058500

[18] Melih Kandemir, Veli-Matti Saarinen, and Samuel Kaski. 2010. Inferring object relevance from gaze in dynamic scenes. In Symposium on Eye-Tracking Research \& Applications (ETRA '10). ACM, 105-108. https://doi.org/10.1145/1743666.1743692

[19] László Kozma, Arto Klami, and Samuel Kaski. 2009. GaZIR: Gaze-based zooming interface for image retrieval. In International Conference on Multimodal Interfaces (ICMI-MLMI '09). 305-312. https://doi.org/10.1145/1647314.1647379

[20] Greg Linden, Brent Smith, and Jeremy York. 2003. Amazon.com recommendations: Item-to-item collaborative filtering. IEEE Internet Computing 7, 1 (2003), 76-80. https://doi.org/10.1109/MIC.2003.1167344 arXiv:69

[21] Ross G. Macdonald and Benjamin W. Tatler. 2018. Gaze in a real-world social interaction: A dual eye-tracking study. Quarterly fournal of Experimental Psychology 71, 10 (2018), 2162-2173. https://doi.org/10.1177/1747021817739221

[22] Yasuto Nakanishi, Takashi Fujii, Kotaro Kiatjima, Yoichi Sato, and Hideki Koike. 2002. Vision-based face tracking system for large displays. Lecture Notes in Computer Science 2498 (2002), 152-159. https://doi.org/10.1007/3-540-45809-3_11

[23] Christina Ohm, Manuel Müller, Bernd Ludwig, and Stefan Bienk. 2014. Where is the landmark? Eye tracking studies in large-scale indoor environments. In CEUR Workshop (ET4S'14). Vienna, Austria, 47-51.

[24] Tobias Otterbring, Erik Wästlund, and Anders Gustafsson. 2016. Eye-tracking customers' visual attention in the wild: Dynamic gaze behavior moderates the effect of store familiarity on navigational fluency. fournal of Retailing and Consumer Services 28 (2016), 165-170. https://doi.org/10.1016/j.jretconser.2015. 09.004

[25] Tobias Otterbring, Erik Wästlund, Anders Gustafsson, and Poja Shams. 2014 Vision (im)possible? The effects of in-store signage on customers' visual attention Journal of Retailing and Consumer Services 21, 5 (2014), 676-684. https://doi.org/ 10.1016/j.jretconser.2014.05.002
[26] Ken Pfeuffer, Melodie Vidal, Jayson Turner, Andreas Bulling, and Hans Gellersen. 2013. Pursuit calibration: Making gaze calibration less tedious and more flexible. In 26th annual ACM symposium on User interface software and technology (UIST '13). ACM, St. Andrews, UK, 261-269. https://doi.org/10.1145/2501988.2501998

[27] Pernilla Qvarfordt and Shumin Zhai. 2005. Conversing With the User Based on Eye-gaze Patterns. In Proceedings of the SIGCHI conference on Human factors in computing systems (CHI '05). 221-230. https://doi.org/10.1145/1054972.1055004

[28] Patrick Renner, Nico Lüdike, Jens Wittrowski, and Thies Pfeiffer. 2011. Towards continuous gaze-based interaction in 3D environments - unobtrusive calibration and accuracy monitoring. In Virtuelle \& Erweiterte Realität. Shaker Verlag, Bonn, Germany, 13-24.

[29] Javier San Agustin, John Paulin Hansen, and Martin Tall. 2010. Gaze-based interaction with public displays using off-the-shelf components. In 12th ACM international conference adjunct papers on Ubiquitous computing (Ubicomp'10). ACM, 377-378. https://doi.org/10.1145/1864431.1864444

[30] India Starker and Richard A. Bolt. 1990. A Gaze-Responsive Self-Disclosing Display. In SIGCHI Conference on Human Factors in Computing Systems Empowering People (CHI '90). ACM, 3-10. https://doi.org/10.1145/97243.97245

[31] Statista. 2020. Volume of data/ information created worldwide from 2010 to 2024. https://www.statista.com/statistics/871513/worldwide-data-created/

[32] Yusuke Sugano, Yasuyuki Matsushita, and Yoichi Sato. 2013. Appearance-based gaze estimation using visual saliency. IEEE Transactions on Pattern Analysis and Machine Intelligence 35, 2 (2013), 329-341. https://doi.org/10.1109/TPAMI.2012. 101

[33] Yusuke Sugano, Yasuyuki Matsushita, Yoichi Sato, and Hideki Koike. 2008. An incremental learning method for unconstrained gaze estimation. Lecture Notes in Computer Science 5304 LNCS, PART 3 (2008), 656-667. https://doi.org/10.1007/ 978-3-540-88690-7-49

[34] Roel Vertegaal, Jeffrey S. Shell, Daniel Chen, and Aadil Mamuji. 2006. Designing for augmented attention: Towards a framework for attentive user interfaces. Computers in Human Behavior 22, 4 (2006), 771-789. https://doi.org/10.1016/j. chb.2005.12.012

[35] Tore Vesterby, Jonas C. Voss, John Paulin Hansen, Arne John Glenstrup, Dan Witzner Hansen, and Mark Rudolph. 2005. Gaze-Guided Viewing of Interactive Movies. Digital Creativity 16, 4 (2005), 193-204. https://doi.org/10.1080/ 14626260500476523

[36] Kang Wang, Shen Wang, and Qiang Ji. 2016. Deep eye fixation map learning for calibration-free eye gaze tracking. In Eye Tracking Research and Applications Symposium (ETRA'16). ACM, Charleston, SC, USA, 47-55. https://doi.org/10. $1145 / 2857491.2857515$

[37] Erik Wästlund, Tobias Otterbring, Anders Gustafsson, and Poja Shams. 2015. Heuristics and resource depletion: Eye-tracking customers' in situ gaze behavior in the field. Journal of Business Research 68, 1 (2015), 95-101. https://doi.org/10. 1016/j.jbusres.2014.05.001

[38] Songhua Xu, Hao Jiang, and Francis C.M. Lau. 2008. Personalized Online Document, Image and Video Recommendation via Commodity Eye-tracking. In Proceedings of the 2008 ACM Conference on Recommender Systems (RecSys '08). 83-90. https://doi.org/10.1145/1454008.1454023 arXiv:1510.03706 